\title{
Blue Compact Dwarf Galaxies: Laboratories for probing the Primordial Universe
}

\author{
Trinh X. Thuan \\ University of Virginia, Astronomy Department \\ P.O. Box 400325, Charlottesville, VA 22904-4325, USA \\ email: txt@virginia.edu
}

\begin{abstract}
Blue Compact Dwarf (BCD) galaxies are the most metal-deficient star-forming galaxies known in the universe, with metallicities ranging from $1 / 40$ to $1 / 3$ that of the Sun. I review how they constitute excellent nearby laboratories for studying big bang nucleosynthesis and star formation and galaxy evolution processes in a nearly primordial environment.
\end{abstract}

Keywords. galaxies: dwarf, galaxies: abundances, galaxies: ISM, galaxies: star clusters, galaxies: individual (I Zw 18), galaxies: individual (SBS 0335-052), stars: winds, stars: outflows, stars: Wolf-Rayet

\section{Blue Compact Dwarfs and Galaxy Formation}

Galaxy formation is one of the most fundamental problems in astrophysics. To understand how galaxies form, we need to unravel how stars form from the primordial gas and how the first stars interact with their surrounding environments. While much progress has been made in finding large populations of galaxies at high redshifts $(z \geqslant 2)$, truly young galaxies in the process of forming remain elusive in the distant universe. The spectra of those far-away galaxies generally indicate the presence of a substantial amount of heavy elements, implying previous star formation and metal enrichment (Shapley et al. 2004). Instead of focussing on high-redshift galaxies, another approach is to study the properties of the massive stellar populations and their interaction with the ambient interstellar medium (ISM) in a class of nearby metal-deficient dwarf galaxies, called Blue Compact Dwarf (BCD) galaxies, which are the least chemically evolved star-forming galaxies known in the universe. These galaxies have an oxygen abundance in the range $12+\log (\mathrm{O} / \mathrm{H})=7.1-8.3$, i.e. $1 / 40-1 / 3$ that of the Sun if the solar abundance of Asplund et al. $(2005), 12+\log (\mathrm{O} / \mathrm{H})_{\odot}=8.7$, is adopted. Thus, the massive stellar populations of BCDs have properties intermediate between those of massive stars in solar-metallicity galaxies such as the Milky Way and those of the first stars. BCDs constitute then excellent nearby laboratories for studying physical processes of galaxy and star formation and chemical enrichment processes in environments that are sometimes much more pristine than those in known high-redshift galaxies. The proximity of BCDs allows studies of their structure, metal content, and stellar populations with a sensitivity, precision, and spatial resolution that faint distant high-redshift galaxies do not allow. In the hierarchical model of galaxy formation, large galaxies result from the merging of dwarf galaxies which are the first structures to collapse and form stars. These building-block galaxies are too faint and small to be studied at high redshifts, while we stand a much better chance of understanding them with local BCDs.

Studies of these very chemically unevolved galaxies will also shed light on galaxy formation theories. Cold Dark Matter (CDM) models predict that low-mass dwarf galaxies could still be forming at the present epoch because they originate from density 
fluctuations considerably smaller and less dense than those giving rise to the giant ones. Thus, if it could be shown that the most chemically unevolved BCDs are also young, i.e. their first stars did not form until $\leqslant 1$ Gyr ago, then the existence of young dwarf galaxies in the local universe will put strong constraints on the primordial density fluctuation spectrum. A key issue in galaxy formation studies is the observed anti-correlation between the stellar mass of a galaxy and the formation epoch of the stars in it, which is often referred to as "downsizing" (Neistein et al. 2006). In the downsizing scenario, massive galaxies are the first to form stars at a high rate, while dwarf galaxies can remain without forming stars and be chemically unevolved for nearly a Hubble time.

\section{A Brief History}

Extragalactic very metal-deficient systems were first discussed by Sargent \& Searle (1970) and Searle \& Sargent (1972). Carrying out a spectroscopic survey of a sample of galaxies selected by Zwicky (1971) on the Palomar Sky Survey photographic plates to have a very compact appearance, the pair came across two very interesting objects, I Zw 18 and II Zw 40. Because the spectra of the two galaxies are "strikingly different from those of galaxies of the Hubble sequence" and resemble those of $\mathrm{H}$ II regions in the Galaxy and the Large Magellanic Cloud, Sargent \& Searle (1970) dubbed them "Isolated extragalactic H II regions". Abundance measurements show both systems to be metaldeficient (about 1/40 solar for I Zw 18 and 1/4 solar for II Zw 40). Because of their low metallicities (more extreme than those of H II regions in the outskirts of spiral galaxies), Searle \& Sargent (1972) argued that these galaxies could be either young, in the sense that the bulk of their star formation has occurred in recent times, or that they could be older systems with star formation in them occurring in intense bursts separated by long quiescent periods. Nearly a decade later, Thuan \& Martin (1981) showed that I Zw 18 and II Zw 40 are part of a general class of dwarf extragalactic systems undergoing intense bursts of star formation, producing young massive blue stars in a localized compact region, which they called Blue Compact Dwarf (BCD) galaxies. Thuan \& Martin (1981) defined a BCD by the following criteria: 1) it has a low luminosity $\left(\mathrm{M}_{B} \geqslant-18 ; 2\right)$ its optical spectrum exhibits strong narrow emission lines superposed on a blue continuum, similar to that of an $\mathrm{H}$ II region; and 3$)$ it has a compact appearance $\left(\mathrm{D}_{25}=1-2 \mathrm{Kpc}\right)$ and a high mean surface brightness $\left(\mathrm{S}_{B} \leqslant 22 \mathrm{mag} \operatorname{arcsec}^{-2}\right)$. Criterion 1$)$ ensures that the object is a dwarf galaxy. It excludes more massive emission-line galaxies with density waves such as Seyfert galaxies. The dwarf criterion also ensures that the BCD has a low heavy-element abundance. Later, Sandage \& Binggeli (1984) extended their dwarf classification system to include BCDs, but their connection with the other types of dwarf galaxies (the dwarf ellipticals and the dwarf magellanic irregulars) is still not yet clear. BCDs have also been sometimes called "H II galaxies" although this term refers to a larger class of emission-line galaxies that include also luminous high-metallicity objects with spiral structure. While on the Palomar Sky Survey plates, the BCDs are "almost stellar in appearance, with no obvious underlying galaxy", later CCD surveys showed them to have nearly always an underlying more extended low-surface-brightness (LSB) component on which are superposed the high-surface brightness compact star-forming regions. The LSB component is redder than the star-forming regions, indicative of an older stellar population. Loose \& Thuan (1986) have proposed a morphological classification scheme for BCDs based on the morphology of both the star-forming regions and of the underlying component. They distinguish four main types of BCDs: 1) the iE BCDs which show a complex structure with several centers of star formation and irregular (i) isophotes in the central regions superposed on a LSB component with elliptical (E) isophotes. They are 
by far the most common type of BCDs; 2) the $\mathrm{nE}$ galaxies with a nuclear (n) star-forming region at the center of a LSB elliptical (E) component; 3) the iI BCDs with irregular (i) star-forming regions superposed on a LSB component with irregular (I) outer isophotes. An interesting subset of iI galaxies are "cometary" galaxies where the star-forming region constitutes the head of the comet and the LSB component the tail; and 4) the i0 galaxies which have irregular (i) star-forming regions but no (0) evident extended underlying older stellar population. The two extremely metal-deficient BCDs discussed below as the prototypes of the two main modes of star formation in BCDs, I Zw 18 and SBS 0335052, are both of type i0. Fig. 1a shows a HST/ACS picture of I Zw 18 (Izotov \& Thuan 2004). It possesses two star-forming regions in the main body, the brighter northwest (NW) and the fainter southeast (SE) components, separated by 8". There is another blue irregular star-forming region 22 " NW of the main body, called the C component. An interferometric H I map of I Zw 18 by van Zee et al. (1998) shows that the C component is embedded within a common $\mathrm{H}$ I envelope with the main body (Fig. 3a). Fig. 1b shows a HST/ACS UV picture of SBS 0335-052E (Thuan \& Izotov 2005). The star formation in SBS 0335-052E occurs mainly in six superstar clusters (SSCs) with ages $\leqslant 25 \mathrm{Myr}$, within a region of 2 " or $520 \mathrm{pc}$ in size. Dust is clearly present and mixed spatially with the SSCs. The SSCs are roughly aligned in the SE-NW direction, and there is a systematic increase in reddening of the clusters away from the brightest one. The observed color dependence on position may be the combined effects of differential extinction by dust and color evolution with time due to sequential propagating star formation. There is a supershell of radius $380 \mathrm{pc}$, delineating a large supernova cavity (Thuan et al. 1997). In contrast to other BCDs, both IZw 18 and SBS 0335-052E do not show evidence of an extended low-surface-brightness underlying component of red old stars.

\section{The most metal-deficient BCDs known and the metallicity floor}

Extremely metal-deficient emission-line galaxies at low redshift are very rare. For more than three decades, I Zw 18 held the record as the most metal-deficient emission-line
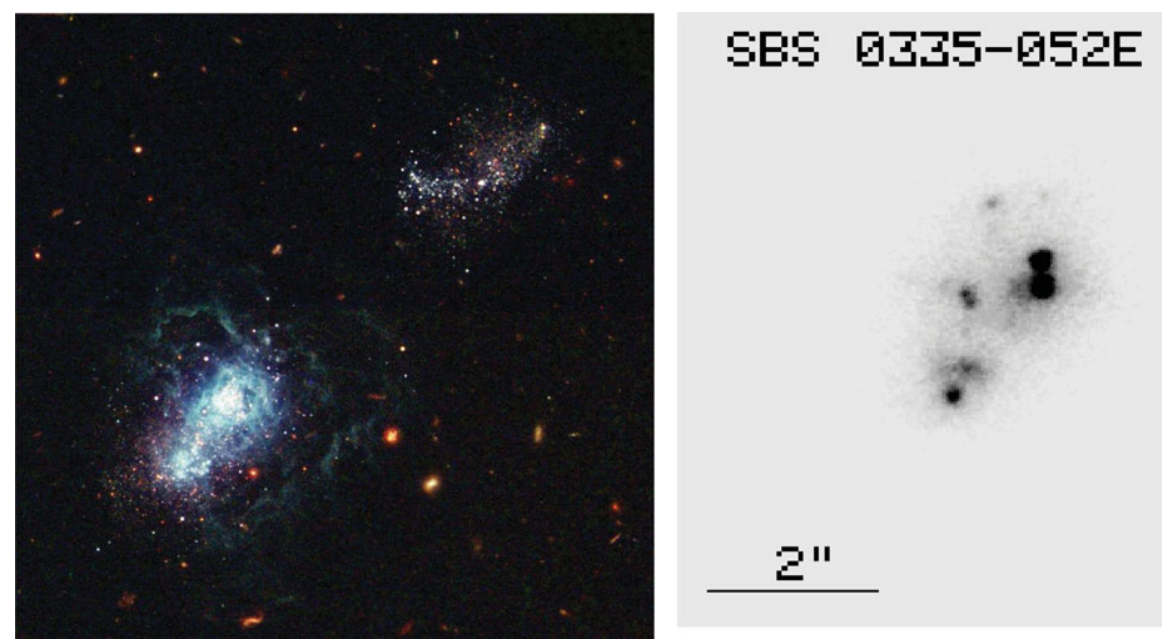

Figure 1. a) HST/ACS V image of I Zw 18 (Izotov \& Thuan 2004). (b) HST/ACS UV image of SBS 0335-052 (Thuan \& Izotov 2005). North is up and east is to the left. Large supershells of ionized gas are seen delineating supernova cavities in both I Zw 18 and SBS 0335-052. However, in both BCDs, no extended low surface brightness underlying component of red old stars is visible. 
galaxy known, with an oxygen abundance $12+\log (\mathrm{O} / \mathrm{H})=7.17 \pm 0.01$ in its $\mathrm{NW}$ component and $7.22 \pm 0.02$ in its SE component (Thuan \& Izotov 2005). That it was one of the first BCDs discovered (Sargent \& Searle 1970) was just sheer beginner's luck. During the two decades that followed, many surveys have been carried out to search for other I Zw 18-like galaxies, without much success. Only in 1990, did Izotov et al. (1990) discover the BCD SBS 0335-052E with an oxygen abundance comparable to that of I Zw 18, $12+\log (\mathrm{O} / \mathrm{H})=7.31 \pm 0.01$ (Thuan \& Izotov 2005). Only very recently has I Zw 18 been displaced by the BCD SBS 0335-052W. The latter was discovered when SBS 0335-052E was mapped in the H I line with the VLA (Pustilnik et al. 2001). The VLA map showed that a huge $\mathrm{H}$ I complex is associated with SBS 0335-052E, having an overall size of about 66 by $22 \mathrm{kpc}$ (Fig. 3b). Two prominent, slightly resolved H I peaks are seen in the integrated H I map, separated in the east-west direction by $22 \mathrm{kpc}$ (84"). While the eastern peak is coincident with SBS 0335-052E, the western peak is associated with another faint BCD, SBS 0335-052W. Izotov et al. (2005) have determined its oxygen abundance to be $12+\log (\mathrm{O} / \mathrm{H})=7.12 \pm 0.03$, making it the emission-line galaxy with the lowest metallicity now known in the local universe. More recently, Pustilnik et al. (2005) have drawn attention to another extremely metal-deficient dwarf galaxy, DDO 68 with $12+\log (\mathrm{O} / \mathrm{H})=7.14 \pm 0.03$ (Izotov \& Thuan 2007).

Can we find more extremely low-metallicity galaxies? We stand a better chance of finding them in very large spectroscopic surveys. One of the best surveys suitable for such a search is the Sloan Digital Sky Survey (SDSS) which include the spectra of a million galaxies. The first three releases yielded no extremely metal-deficient objects. The lowestmetallicity emission-line galaxies found in these early releases all have $12+\log (\mathrm{O} / \mathrm{H}) \geqslant 7.4$ (e.g. Kniazev et al. 2003). Recently, Izotov et al. (2006) and Izotov \& Thuan (2007) have started a spectroscopic survey of SDSS galaxies selected to have [O III] $\lambda 4959 / \mathrm{H} \beta \leqslant 1$ and $[\mathrm{N}$ II $] \lambda 6583 / \mathrm{H} \beta \leqslant 0.05$. These spectral properties uniquely select out low-metallicity dwarfs, since no other type of galaxy possesses them. Those authors discovered two

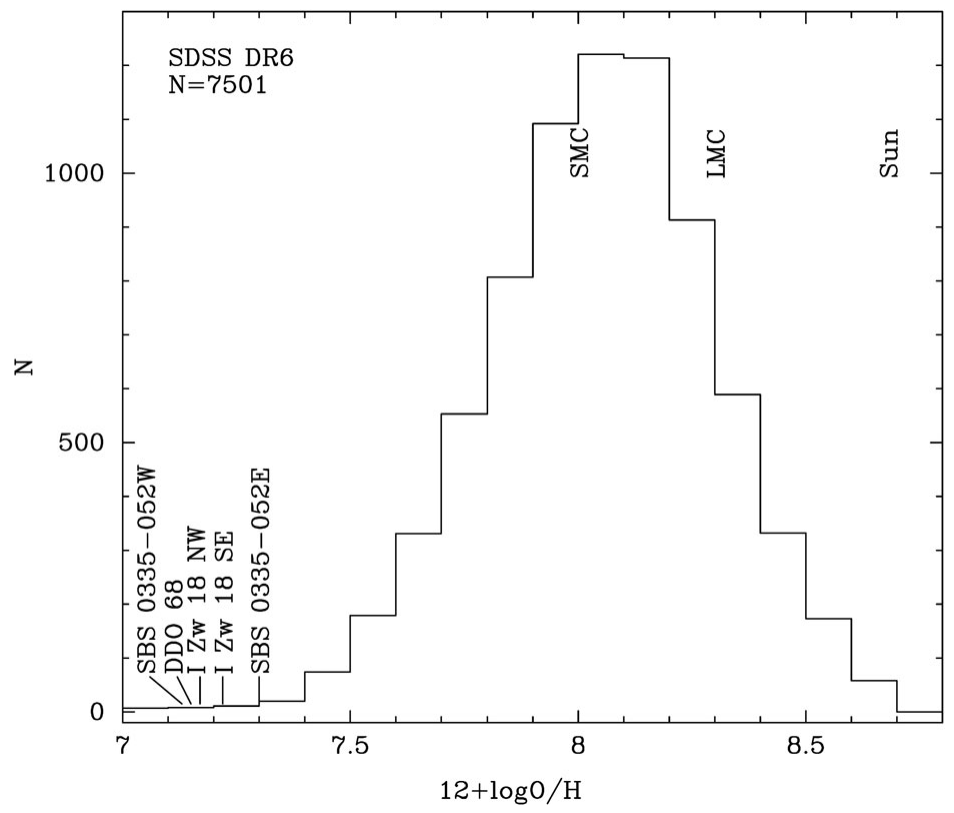

Figure 2. The metallicity distribution of all galaxies in the SDSS Data Release 6 with a H II region-like spectrum and a detected [O III] $\lambda 4363$ line. 
new extremely metal-deficient galaxies, J0113+0052 (UGC 772) with $12+\log (\mathrm{O} / \mathrm{H})=$ $7.24 \pm 0.05$, and $\mathrm{J} 2104-0035$ with $12+\log (\mathrm{O} / \mathrm{H})=7.26 \pm 0.03$. All other objects have $12+\log (\mathrm{O} / \mathrm{H})$ between 7.3 and 7.8. Thus, at the present time, the 7 most metal-deficient objects known in the local universe, in order of increasing metallicity (see also Guseva in these proceedings), are: SBS 0335-052W, DDO 68, I Zw 18 NW, I Zw 18 SE, J0113+0052, J2104-0035 and SBS 0335-052E. I Zw 18 has lost its title of "most metal-deficient starforming dwarf" it held for some 35 years, to be relegated to third place. Fig. 2 shows the metallicity distribution of the 7501 emission-line galaxies selected from the SDSS Data Release 6 to have a H II region-like spectrum and a detected [O III] $\lambda 4363$ line (for accurate abundance determination). It is clear that the low-metallicity end of the distribution is filling in (compare with the list of metal-poor galaxies given 8 years ago by Kunth \& Östlin 2000). However, despite much effort, no H II region with $12+\log (\mathrm{O} / \mathrm{H}) \leqslant 7.1$ has been found. The existing data appear to suggest the existence of an oxygen abundance floor for the ionized gas of BCDs.

\section{Is the neutral gas in BCDs primordial?}

How about the neutral gas component? BCDs all possess an important neutral gas component to feed the star formation (e.g. Thuan \& Martin 1981, Thuan et al. 1999b). One may suppose that, if the BCD is young, i.e. undergoing now one of its first bursts of star formation, then the $\mathrm{H}$ I gas in BCDs may be primordial, devoid of any heavy element. Is this the case? The advent of FUSE has allowed to attack that problem. Using the $\mathrm{H}$ II regions in the BCD as sources of UV light shining through the $\mathrm{H}$ I envelope (Fig. 3), FUSE can obtain absorption spectra which can be used to determine the heavy element abundances in the neutral gas of the BCD. Thus far some 8 BCDs and dwarf irregular galaxies have been observed by FUSE (Lebouteiller, these proceedings). The general result is that there appears to be also a metallicity floor for the H I gas of BCDs, at about the same level as for the ionized gas. The oxygen abundance of the neutral gas appears to be constant at $12+\log (\mathrm{O} / \mathrm{H})_{\text {neutral }} \sim 7.0$, for those BCDs with $12+\log (\mathrm{O} / \mathrm{H})_{\text {ionized }} \leqslant 8$, increasing with the abundance of the ionized gas in BCDs with higher metallicities (Lebouteiller). This supports the suggestion of Thuan et al. (2005) that the matter from which dwarf emission-line galaxies formed was preenriched to a common level of about $2 \%$ of the abundance of the Sun, possibly by Population III stars. This idea appears to be also supported by the oxygen abundances $12+\log (\mathrm{O} / \mathrm{H})$
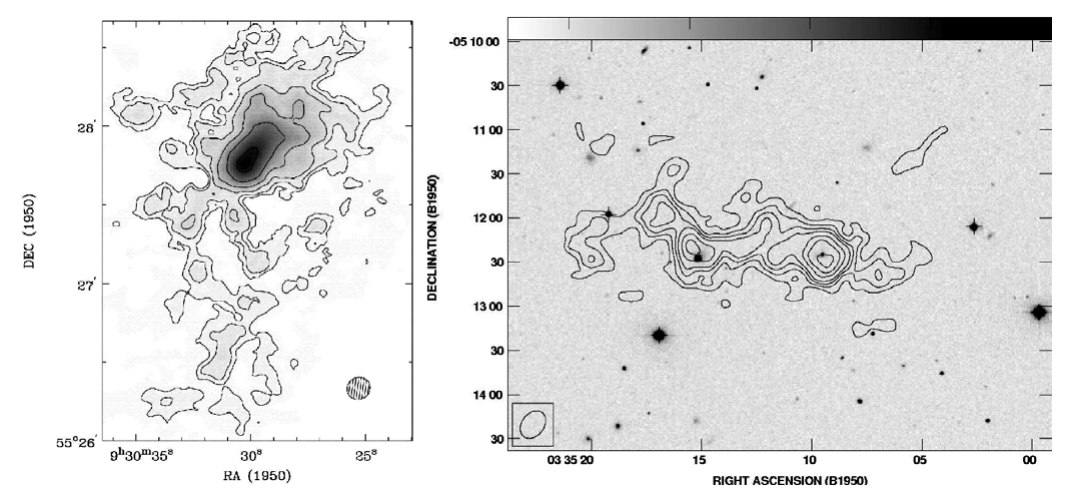

Figure 3. VLA H I maps of: a) I Zw 18 (van Zee et al. 1998). The main body and the C component are located at the H I peak; b) the SBS0335-052 complex (Pustilnik et al. 2001). SBS 0335-052E and SBS 0335-052W are located at the two H I peaks. 
between 6.7 and 7.6 derived by e.g. Telfer et al. (2002) for the intergalactic medium, using ultraviolet absorption lines in Ly $\alpha$ absorbers.

\section{The hot gas component}

The generation of large amounts of hot (a few times $10^{6} \mathrm{~K}$ ) and rarefied gas, resulting from the injection of energy and momentum into the cold ambient interstellar medium by stellar winds from massive stars and supernovae (SNe), has important consequences for the subsequent dynamical and chemical evolution of BCDs. The starburst events that are responsible for the hot X-ray emitting gas last about $10^{7} \mathrm{yr}$ each, and are separated by long quiescent periods of more than 1-2 Gyr (Fanelli et al. 1988). Tenorio-Tagle (1996) has proposed a "galactic fountain" scenario in which the SNe nucleosynthesis products are driven to high galactic latitudes in a hot phase before cooling down and falling back onto the stellar body as molecular droplets. This naturally explains the uniform abundances that are observed in separate $\mathrm{H}$ II regions within the same BCD. To test this galactic fountain scenario, Thuan et al. (2004) have obtained Chandra X-ray observations of three of the most metal-deficient BCDs known, SBS 0335-052E, SBS 0335-052W, and I Zw 18. X-ray emission is indeed detected from all three BCDs. The $0.5-10.0 \mathrm{keV}$ luminosities of these objects are in the range $1.3-8.5 \times 10^{39} \mathrm{ergs} \mathrm{s}^{-1}$. But more than $80 \%$ of the X-ray emission comes from point sources, which Thuan et al. (2004) attribute to ultraluminous high-mass X-ray binaries. There are hints of faint extended diffuse X-ray emission in SBS 0335052E and I Zw 18, probably associated with the superbubbles visible in both BCDs. However, the X-ray images do not show the hot gas breaking out from the stellar body, as predicted by the galactic fountain scenario. These observations also constrain chemical evolution models of dwarf galaxies which rule out closed-box models and require gas infall or loss of metals by galactic winds to account for the observed chemical abundances (Matteucci, these proceedings). There is no evident sign of galactic winds in the X-ray maps of SBS 0335-052E, SBS 0335-052W, and I Zw 18.

\section{The "active" versus "passive" mode of star formation in BCDs}

We now discuss star formation in BCDs. Based on the detailed investigation of two of the most metal-deficient and most studied BCDs, SBS 0335052E and I Zw 18, star formation in BCDs appears to occur in two very different modes: an "active" mode (we follow the terminology of Hirashita \& Hunt 2004) of which SBS 0335052E is the prototype, and a "passive" mode, of which I Zw 18 is the prototype. The active mode is characterized by super-star cluster (SSC) formation, a high star formation rate (SFR), a very compact size, hot dust, and significant amounts of molecules such as $\mathrm{H}_{2}$. On the other hand, the passive mode is characterized by an absence of SSCs, a low SFR, a larger size, cooler dust and no significant amount of $\mathrm{H}_{2}$. The observed characteristics of SBS 0335052E and I Zw 18 are summarized in Table 1. Clearly, metallicity is not the distinguishing factor between these two modes of star formation since both BCDs have similarly low heavy element abundance. Hirashita \& Hunt (2004) have suggested that the difference between the two modes can be understood through a difference in size and density of the star-forming regions. The active mode occurs in regions that are compact and dense. On the other hand, the passive mode occurs in regions that are diffuse (with radius $\geqslant 100 \mathrm{pc}$ ) and less dense.

Indeed, the SSCs in SBS 0335-052E are compact ( $\leqslant 25 \mathrm{pc})$ and dense $\left(\sim 100 \mathrm{pc}^{-2}\right)$ (Thuan et al. 1997), while the star clusters in I Zw 18 are instead ordinary young clusters, $\geqslant 300 \mathrm{pc}$ in size and $\leqslant 0.02 \mathrm{pc}^{-2}$, comparable to those in M 101, and not as extreme as 
Table 1. Active versus Passive Star Formation in SBS 0335-052E and I Zw 18

\begin{tabular}{|c|c|}
\hline SBS 0335-052E & I $\mathrm{Zw}_{\mathrm{w}} 18$ \\
\hline $\begin{array}{l}\text { Super star clusters }(\mathrm{SSCs}) \\
\mathrm{n}_{e}(\mathrm{~S} \text { II })=390 \mathrm{~cm}^{-3} \\
\mathrm{n}_{e}(\text { radio })=2000 \mathrm{~cm}^{-3} \\
\text { Compact radio source: } 17 \mathrm{pc} \\
\text { Total SFR }(\text { radio, IR })=0.7-0.8 \mathrm{M}_{\odot} \mathrm{yr}^{-1} \\
\text { Thermal emission: } 9000 \mathrm{O} 7 \mathrm{~V} \text { stars } \\
\text { High-ionization lines: }[\mathrm{Ne} \mathrm{V}](7 \mathrm{Ryd}) \\
\mathrm{H}_{2} \\
\text { Very dusty : } \mathrm{M}_{\text {dust }}=1.5-6 \times 10^{3} \mathrm{M}_{\odot} \\
\text { Continuum MIR peak: } \sim 28 \mu \mathrm{m}\end{array}$ & $\begin{array}{l}\text { No SSCs } \\
\mathrm{n}_{e}(\mathrm{~S} \text { II }) \leqslant 100 \mathrm{~cm}^{-3} \\
\text { less compact } \\
\text { Total SFR (radio) }=0.1 \mathrm{M}_{\odot} \mathrm{yr}^{-1} \\
\text { Thermal emission: } 1200 \mathrm{O} 7 \mathrm{~V} \text { stars } \\
\text { No high-ionization lines } \\
\text { No } \mathrm{H}_{2} \\
\text { Not so dusty } \\
\text { Continuum peaks longwards of } 70 \mu \mathrm{m}\end{array}$ \\
\hline
\end{tabular}

those in 30 Doradus. The electron density of SBS 0335-052E as derived from the [S II] $\lambda \lambda 6717,6731$ optical emission line ratio is equal to $390 \mathrm{~cm}^{-3}$, while that derived for I $\mathrm{Zw} 18$ is $\leqslant 100 \mathrm{~cm}^{-3}$. The electron density as derived from the radio emission in SBS 0335-052E, which probes more extincted star-forming regions, is even higher. The radio emission of SBS 0335-052E shows significant free-free absorption at $1.4 \mathrm{GHz}$ (Hunt et al. 2004), while there is no evidence for such a spectral turnover in I Zw 18 (Hunt et al. 2005). The inferred density for SBS 0335-052E from the radio free-free absorption is $\sim 2000 \mathrm{~cm}^{-3}$. The radio emission of SBS 0335-052E comes from an extremely compact region (its diameter is $17 \mathrm{pc}$ ), while the radio emission in $\mathrm{I} \mathrm{Zw} 18$ is diffuse and extended, despite a similar global thermal/non-thermal mix (at $4.8 \mathrm{GHz}$ : 40/60 in I Zw 18 and 30/70 in SBS 0335-052E). The radio luminosity of $\mathrm{I} \mathrm{Zw} 18$ is considerably smaller than that of SBS 0335-052E; the non-thermal radio luminosity of $\mathrm{I} \mathrm{Zw} 18$ is only $\sim 5 \%$, and its thermal component only about $25 \%$ of that of SBS 0335-052E. The total SFR in I Zw 18 as derived from the radio emission is $\sim 0.1 \mathrm{M}_{\odot} \mathrm{yr}^{-1}$, nearly one order of magnitude lower than that the one in SBS 0335-052E.

Before the mid-infrared (MIR) ISO observations of SBS 0335-052E by Thuan et al. (1999), it was generally thought that low-metallicity BCDs would not contain much dust. Thuan et al. (1999) found that although SBS 0335-052E is one of the most metaldeficient BCDs known (4\% solar), it is unexpectedly bright in the MIR range: as much as $75 \%$ of the total luminosity of SBS $0335-052 \mathrm{E}$ comes as MIR radiation. Later Spitzer observations by Houck et al. (2004) have shown that the total dust mass in SBS 0335$052 \mathrm{E}$ is $\sim 1.5 \times 10^{3} \mathrm{M}_{\odot}$. The MIR spectrum peaks around $28 \mu \mathrm{m}$, which indicates that there is very little cold dust in SBS 0335-052E. Contrary to the spectra of typical solar metallicity starburst galaxies, the spectrum of SBS 0335-052E does not show polycyclic aromatic hydrocarbon $(\mathrm{PAH})$ emission features. This is due to the very low metallicity of the BCD (the PAH features are weaker at lower metallicities, see e.g. Wu et al. 2006) but also to its very high UV luminosity density which destroys PAHs. On the other hand, the continuum MIR emission of I Zw 18 has a much steeper slope, more like that of a starburst galaxy. It peaks longward of $70 \mu \mathrm{m}$, indicating a significant amount of cold dust in I Zw 18. As for SBS 0335-052E, the MIR spectrum of I Zw 18 does not show any PAH feature, because of its very low metallicity (Wu et al. 2007).

Concerning the molecular content, FUSE did not detect any diffuse $\mathrm{H}_{2}$ in either SBS0335-052E (Thuan et al. 2005) or I Zw 18 (Vidal-Madjar et al. 2001). The absence of diffuse $\mathrm{H}_{2}$ is due to the combined effects of a low $\mathrm{H}$ I density in the neutral gas envelope, a large UV flux that destroys $\mathrm{H}_{2}$ molecules and a low metallicity that makes grains on which to form $\mathrm{H}_{2}$ molecules scarce. However Vanzi et al. (2000) did detect several $\mathrm{H}_{2}$ emission lines in their near-infrared spectrum of SBS0335-052E. These lines observed in the $\mathrm{K}$ band are generally consistent, within the errors, with both thermal and fluorescent 
excitation by the strong UV field. This implies that the detected $\mathrm{H}_{2}$ must be clumpy and associated only with the dense star-forming regions. These clumps should be denser than $1000 \mathrm{~cm}^{-3}$ and have a temperature greater than $1000 \mathrm{~K}$ (Thuan et al. 2005).

\section{High-ionization radiation}

There exists also a difference between the active and passsive modes concerning the amount of very hard ionizing radiation in the star-forming regions. SBS 0335-052E contains very hard radiation, with energies in excess of $95 \mathrm{eV}$, while the radiation in I Zw 18 is not as hard. The hardness of the ionizing radiation in BCDs has long been known to increase with decreasing metallicity. This is supported by the fact that the strong nebular emission line He II $\lambda 4686$ is often seen in the spectra of BCDs, with a flux that increases with decreasing metallicity of the ionized gas (Guseva et al. 2000). Besides He II emission, high-ionization emission lines of heavy elements ions are also seen in the spectra of some BCDs, such as the high-ionization emission line [Fe v] $\lambda 4227$ in the BCDs Tol 1214-277 $(12+\log \mathrm{O} / \mathrm{H}=7.55)$ and SBS 0335-052E. The presence of this line, just as that of the He II $\lambda 4686$ line, requires ionizing radiation with photon energies in excess of 4 Rydberg. Later, the $[\mathrm{Ne} \mathrm{v}] \lambda \lambda 3346,3426$ emission lines were detected in the spectra of the BCDs SBS0335-052E, HS0837+4717 $(12+\log \mathrm{O} / \mathrm{H}=7.60)$, Tol 1214-277, and perhaps of Mrk $209(12+\log \mathrm{O} / \mathrm{H}=7.82)$ (Fig. 4a) (Thuan \& Izotov 2005). The existence of these lines requires the presence of hard radiation with photon energies above 7.1 Rydberg, i.e., in the range of soft $\mathrm{X}$-rays. Such very hard ionizing radiation is unexpected: it has been seen before in AGN, but not in star-forming galaxies. By contrast, while the spectrum of I Zw $18 \mathrm{NW}$ does show the He II $\lambda 4686$ line and a hint of the $[\mathrm{Fe}$ V] $\lambda 4227$ line, the $[\mathrm{Ne} \mathrm{v}] \lambda \lambda 3346,3426$ lines are conspicuously absent (Thuan \& Izotov 2005). This means
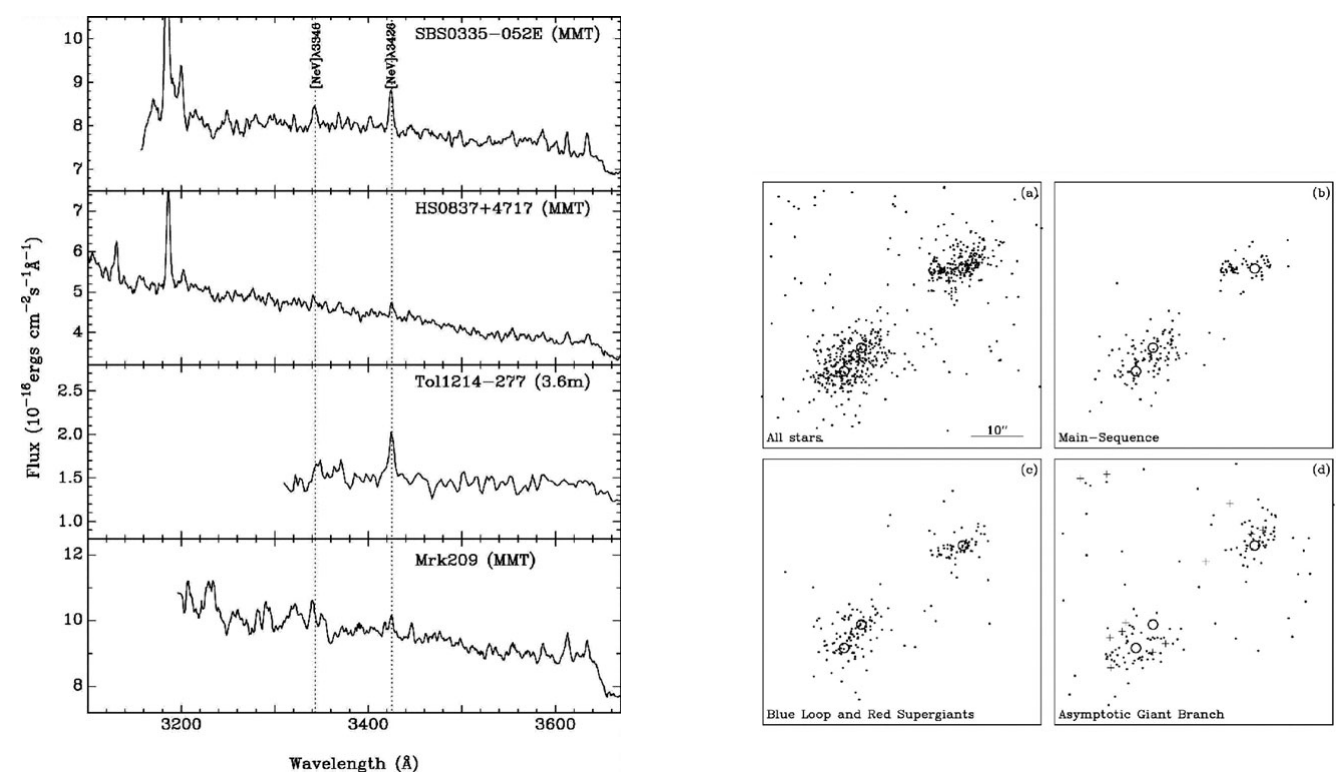

Figure 4. a) Spectra of all known BCDs with detected [Ne v] $\lambda \lambda 3346,3426$ lines. The location of these lines are indicated by dotted vertical lines (Thuan \& Izotov 2005); b) Spatial distributions of (a) stars of all types, (b) MS stars, (c) BL and RSG stars, and (d) AGB stars in I Zw 18 (dots). Open circles show the locations of the NW and SE components in the main body and of the central cluster in the C component (Izotov \& Thuan 2004). 
that I Zw $18 \mathrm{NW}$ contains radiation as hard as 4 Rydberg, but not as hard as $7.1 \mathrm{Ry}-$ dberg, as in SBS 0335-052E. There is a general trend of higher [Ne v], [Fe v] and He II emission at lower metallicities. However metallicity cannot be the only factor which controls the hardness of the radiation, since SBS 0335-052 and I Zw 18 have about the same metallicity.

It is interesting to note that Spitzer IRS spectra of some higher-metallicity BCDs such as Haro $3(12+\log \mathrm{O} / \mathrm{H}=8.34$; Hunt et al. 2006 , these proceedings $)$ and Mrk 996 $(12+\log \mathrm{O} / \mathrm{H}=8.0$; Thuan et al. 2008$)$ show the presence of the [O IV] $\lambda 25.89 \mu \mathrm{m}$ line, with an ionization potential of $54.9 \mathrm{eV}$, just beyond the He II edge at $54.4 \mathrm{eV}$. However, the optical spectra of these objects do not show the presence of the high-ionization [Fe v] $\lambda 4227$ and He II $\lambda 4686$ nebular emission lines which both also have an ionization potential of $54.4 \mathrm{eV}$. This implies that the hard ionizing radiation in these objects comes entirely from a region that is hidden in the optical but seen in the MIR.

What is the origin of the hard ionizing radiation beyond 4 Rydberg, and especially beyond 7 Rydberg? Thuan \& Izotov (2005) found that the hardness of the ionizing radiation with photon energies greater than 4 Rydberg does not generally depend on the burst age as measured by the equivalent width of $\mathrm{H} \beta$. But there is a clear absence of He II emission in very young 3-4 Myr starbursts with $\mathrm{EW}(\mathrm{H} \beta) \geqslant 300 \AA$, implying that the source of ionization with photon energy greater than 4 Rydberg is not massive stars in their main-sequence phase, but in their post-main-sequence phase. Strong He II emission is seen in starbursts with $\operatorname{EW}(\mathrm{H} \beta) \sim 50-300 \AA$, suggesting that less massive stars and their descendants also contribute radiation to ionize helium. However present non-rotating photoionization models of main-sequence stars (such as the Costar models of Schaerer \& de Koter 1997) and of Wolf-Rayet stars (Schaerer \& Vacca 1998) cannot reproduce the observed intensity of the He II emission in very low metallicity BCDs. It would be interesting to see whether the new rotating stellar models by the Geneva group can do a better job. The X-ray luminosity in luminous high-mass X-ray binaries (HMXBs) observed in a few very metal-deficient BCDs, such as SBS 0335-052 (Thuan et al. 2004), can account for their [Ne V] emission. However, the scarce X-ray data do not show a one-to-one correlation between the presence of a HMXB and [Ne V] emission (SBS 0335-052 shows [Ne v] emission, but I Zw 18 does not, although both have HMXBs) so that HMXBs are probably ruled out as the main source of hard ionizing radiation with energy above 7.1 Ryd. Thuan \& Izotov (2005) found that the most likely source of $[\mathrm{Ne} \mathrm{V}]$ emission is probably fast radiative shocks moving with velocities $450 \mathrm{~km} \mathrm{~s}^{-1}$ through a dense ISM with electron number densities of several hundreds $\mathrm{cm}^{-3}$ (Dopita \& Sutherland 1996). These shocks can probably be produced via the evolution of 50-100 $\mathrm{M}_{\odot}$ stars, formed in very compact and dense SSCs in the "active" mode of star formation. These fast radiative shocks are evidenced by broad components in the line profiles of the strong emission lines (Izotov et al. 2007b). However the presence of compact SSCs and broad components is not necessarily accompanied by high-ionization emission. Metallicity appears to play an important role. [ $\mathrm{Ne} \mathrm{V}$ ] emission is detected only in low- metallicity galaxies with $12+\log \mathrm{O} / \mathrm{H} \leqslant 7.8$. In higher-metallicity galaxies, the postshock regions are cooled more efficiently. Therefore, their high-ionization regions are smaller and their emission lower.

\section{The age of $\mathrm{I} \mathrm{Zw} 18$}

We now turn to the question posed more than 3 decades ago by Searle \& Sargent (1972): are the most metal-deficient BCDs young galaxies, i.e. they formed their first stars at a relatively recent time ( $\leqslant 1 \mathrm{Gyr}$ ago), or are they old galaxies which formed most of 
their stars several Gyr ago, in which case the present starburst is just the last episode of a series of such events? For the vast majority of BCDs (more than 99\%), the first alternative is true: their CCD images show clearly an extended low-surface-brightness component indicative of an older stellar population. Photometric studies combined with spectral population synthesis models of a few BCDs give ages of a few Gyr for the older stellar population, considerably smaller than the age of the universe. For example, the study of the two "cometary" BCDs Mrk 59 and Mrk 71 by Noeske et al. (2000) gives an age of $\sim 4$ Gyr for the older stellar population, consistent with the age found by Thuan \& Izotov (2005b) from a HST/WFPC2 color-magnitude diagram study of Mrk 71 (NGC 2366).

However, the most metal-deficient BCDs, those with $12+\log \mathrm{O} / \mathrm{H} \leqslant 7.3$ discussed in Section 3, do not appear to have an extended lower surface brightness component. Of the objects listed in Section 3, only I Zw 18 and SBS0335-052E have been extensively photometrically studied. Photometry combined with spectrophotometric data give ages $\leqslant 1$ Gyr for the compact underlying population of these two objects (Papaderos et al. 1998 for SBS 0335-052E; Papaderos et al. 2002 and Hunt et al. 2003 for I Zw 18). The most direct way to determine the age of a galaxy is by constructing its color-magnitude diagram (CMD). Among the galaxies discussed in Section 3, only I Zw 18 and DDO 68 are near enough to be resolved into stars by HST. Izotov \& Thuan (2004) (hereafter IT04) obtained deep V and I HST/ACS images to construct a CMD of I Zw 18. They did not detect a well developed red giant branch (RGB), which led them to conclude that the age of I Zw 18 is 500 Myr. They also derive a distance to I Zw 18 in the range 12.615 Mpc. Later, Aloisi et al. (2007)(hereafter A07) (see also these proceedings) obtained more ACS data for I Zw 18 and combining with the data of IT04 constructed a deeper CMD of I Zw 18. According to these authors, there is a red giant branch (RGB) in I $\mathrm{Zw} \mathrm{18,} \mathrm{the} \mathrm{tip} \mathrm{of} \mathrm{which} \mathrm{(TRGB)} \mathrm{gives} \mathrm{a} \mathrm{distance} \mathrm{of} 18.2 \mathrm{Mpc}$, in agreement with the distance given by one classical Cepheid they found in the galaxy. Because of the presence of RGB stars, A07 concluded that the age of the oldest stars in I Zw 18 is $\geqslant 1$ Gyr, an age at least twice as large as the age of 0.5 Gyr found by IT04. Despite the work of A07, we feel that the verdict is not in yet concerning the age of $\mathrm{I} \mathrm{Zw}$ 18. Derived ages depend on theoretical isochrones, the location of which in the CMD depends on the adopted distance to the galaxy. A distance as large as $18.2 \mathrm{Mpc}$ for I Zw 18 poses several problems which need to be understood before we can accept it. First, the distance determination rests on a single classical Cepheid, and the period-luminosity of Cepheids at low metallicities is not well-known. Second, at the distance of $18.2 \mathrm{Mpc}$, the $I$ absolute magnitude of the Asymptotic Giant Branch (AGB) stars in I Zw 18 would be brighter by $\sim 0.8 \mathrm{mag}$ than those in other low-metallicity BCDs. The magnitude difference between the TRGB and the AGB stars in I Zw 18 would be some 1.8 mag in the $I$ band instead of $\leqslant 1 \mathrm{mag}$ as in other galaxies. To reconcile the properties of the AGB stars in I Zw 18 with those in other dwarf galaxies, IT04 argued that the distance of I Zw 18 should be close to $15 \mathrm{Mpc}$. Furthermore, the determination of the TRGB by A07 relies on a very small number of stars. Even if I Zw 18 contains RGB stars, their number is unusually small as compared to other BCDs with $12+\log \mathrm{O} / \mathrm{H} \geqslant 7.3$, which possess well-populated RGBs and well-defined TRGBs.

Finally, the spatial distribution of stars with different ages in I $\mathrm{Zw} 18$ is not easily understood if the latter is an old galaxy. Fig. 4b shows that the older AGB stars are not more spread out spatially than the younger main-sequence (MS), blue loop (BL) and red supergiant (RSG) stars. Such a distribution is drastically different from the situation in other BCDs, where the old AGB and RGB stars are distributed over a considerably larger area as compared to younger stars because of diffusion and relaxation processes of stellar 
ensembles. If anything, the reverse appears to be true in I $\mathrm{Zw} 18$ : the MS and BL + RSG stars are distributed over a larger area around the main body as compared to the AGB stars. This suggests that the star formation in the past responsible for the AGB stars was more concentrated in the main body, while recent star formation responsible for the MS and BL + RSG stars is more spread out. I Zw 18 appears to be a young galaxy in the process of forming from the inside out.

\section{The Primordial ${ }^{4} \mathrm{He}$ Abundance}

Because they are the least chemically evolved star-forming objects known in the universe, BCDs contain very little helium manufactured by stars after the big bang. They are thus excellent objects in which to measure the primordial ${ }^{4} \mathrm{He}$ abundance. In the standard theory of big bang nucleosynthesis ( $\mathrm{SBBN})$, given the number of light neutrino species, the abundances of light elements depend only on one cosmological parameter, the baryon density parameter, the present ratio of the baryon mass density to the critical density of the universe. This means that accurate measurements of the primordial abundances of each of the four light elements can provide, in principle, a direct measurement of the baryonic mass density. Because of the strong dependence of its abundance on baryonic mass density, deuterium has become the baryometer of choice ever since accurate measurement of $\mathrm{D} / \mathrm{H}$ in high-redshift low-metallicity QSO Ly $\alpha$ absorption systems became possible. While a single good baryometer like D is sufficient to derive the baryonic mass density from big bang nucleosynthesis, accurate measurements of the primordial abundances of at least two different relic elements are required to check the consistency of SBBN. Among the remaining relic elements, ${ }^{3} \mathrm{He}$ has undergone significant chemical evolution after the big bang, making it difficult to derive its primordial abundance while the derivation of the primordial ${ }^{7} \mathrm{Li}$ abundance in metal-poor halo stars in the Galaxy is beset by difficulties such as the uncertain stellar temperature scale and the temperature structures of the atmospheres of these very cool stars. Thus ${ }^{4} \mathrm{He}$ remains the light element of choice to test SBBN.

For the last decade and a half, I have been involved with my colleague Yuri Izotov in a sustained effort to obtain a precise determination of the primordial He mass fraction $\mathrm{Y}_{p}$. However, to detect small deviations from SBBN (such as deviations from the standard rate of Hubble expansion or a possible asymmetry between the numbers of neutrinos and antineutrinos in the early universe), and make cosmological inferences, $\mathrm{Y}_{p}$ has to be determined to a level of accuracy of less than $1 \%$. Attaining that precision requires many conditions to be met. First, the observational data have to be of excellent quality. This has been the concern of our group. Over the years, we have been obtaining high signal-to-noise ratio spectroscopic data of low-metallicity extragalactic $\mathrm{H}$ II regions, and our sample now includes a total of $86 \mathrm{H}$ II regions in 77 galaxies (Izotov et al. 2007). This constitutes by far the largest sample of high-quality data reduced in a homogeneous way to investigate the problem of the primordial helium abundance. Second, all known systematic effects that may affect the $\mathrm{Y}_{p}$ determination must be taken into account. They include different sets of He I line emissivities and reddening laws, collisional and fluorescent enhancements of He I recombination lines, underlying He I stellar absorption lines, collisional excitation of hydrogen lines, temperature and ionization structure of the $\mathrm{H}$ II region, and deviation of He I and $\mathrm{H}$ emission-line intensities from case B. Using Monte Carlo methods to solve simultaneously for the above systematic effects, Izotov et al. (2007) find that most of the present uncertainty in $\mathrm{Y}_{p}$ comes from the He I emissivities. They find values of $\mathrm{Y}_{p}$ equal to $0.2472 \pm 0.0012$ and $0.2516 \pm 0.0011$ for the 2 sets of He I emissivities currently in the 
literature. The first value agrees well with the value given by SBBN theory, while the second value would imply slight deviations from SBBN.

\section{Acknowledgements}

I wish to thank L. Hunt, S. Madden and R. Schneider for organizing a wonderful conference in an enchanting setting.

\section{References}

Aloisi, A., Clementini, M., \& Tosi, M. et al. 2007, ApJ, 667, L151

Asplund, M., Grevesse, N., \& Sauval, A.J. 2005, in: T.G. Barnes III \& F. N. Bash (eds.), Cosmic Abundances as Records of Stellar Evolution and Nucleosynthesis, ASP Ser. 336, p. 25

Dopita, M. A. \& Sutherland, R. S. 1996, ApJS, 102, 161

Fanelli, M. N., O'Connell, R. W., \& Thuan, T. X. 1988, ApJ, 334, 665

Guseva, N. G., Izotov, Y. I., \& Thuan, T. X. 2000, ApJ, 531, 776

Hirashita, H. \& Hunt, L. K. 2004, A\&A A, 421, 555

Houck, J. R. et al. 2004, ApJS, 154, 211

Hunt, L. K., Thuan, T. X., \& Izotov, Y. I. 2003, ApJ, 588, 281

Hunt, L. K., Dyer, K. K., Thuan, T. X., \& Ulvestad, J. S. 2004, ApJ, 606, 853

Hunt, L. K., Dyer, K. K., \& Thuan, T. X. 2005, A\&A, 436, 837

Hunt, L. K., Thuan, T. X., Sauvage, M., \& Izotov, Y. I. 2006, ApJ, 653, 222

Izotov, Y. I. \& Thuan, T. X. 2004, ApJ, 616, 768

Izotov, Y. I. \& Thuan, T. X. 2007, ApJ, 665, 1115

Izotov, Y. I., Thuan, T. X., \& Guseva, N. G. 2005, ApJ, 632, 210

Izotov, Y. I., Thuan, T. X., \& Stasińska, G. 2007, ApJ, 662, 15

Izotov, Y. I., Thuan, T. X., \& Guseva, N. G. 2007b, ApJ, 671, 1297

Izotov, Y. I., Guseva, N.G., Lipovetsky, V.A., et al. 1990, Nature, 343, 238

Izotov, Y. I., Papaderos, P., Guseva, N. G., Fricke, K. J., \& Thuan, T. X. 2006, A $\& A, 454,137$

Kniazev, A. Y., Grebel, E. K., Hao, L., et al. 2003, ApJ, 593, L73

Kunth, S. \& Östlin, G. 2000, A\&SAR, 10, 1

Loose, H.-H. \& Thuan, T. X. 1986, in: D. Kunth, T.X. Thuan \& J.T.T. Van (eds.), Star-forming dwarf galaxies and related objects, (Gif-sur-Yvette: Editions Frontières), p. 73

Neistein, E., van den Bosch, F. C., \& Dekel, A. 2007, MNRAS, 372, 933

Noeske, K. G., Guseva, N. G., Fricke, K. J. et al. 2000, A\&A, 361, 33

Papaderos, P., Izotov, Y. I., Fricke, K. J. et al. 1998, $A \& A, 338,43$

Papaderos, P., Izotov, Y. I., Thuan, T. X. et al. 2002, A\&A, 393, 461

Pustilnik, S. A., Kniazev, A. Y., \& Pramskij, A. G. 2005, A\&A, 443, 91

Pustilnik, S. A., Brinks, E., Thuan, T. X., Lipovetsky, V. A., \& Izotov, Y. I. 2001, AJ, 121, 1413

Sargent, W. L. W. \& Searle, L. 1970, ApJ, 162, L155

Sandage, A. \& Binggeli, B. 1984, AJ, 89, 919

Schaerer, D. \& de Koter, A. 1997, A\& $A, 322,598$

Schaerer, D. \& Vacca, W. D. 1998, ApJ, 497, 618 “

Searle, L. \& Sargent, W.L.W. 1972, ApJ, 173, 25

Shapley, A. E., Erb, D. K.; Pettini, M, Steidel, C. C., \& Adelberger, K. L. 2004, ApJ, 612, 108

Telfer, R. C., Kriss, G. A., Zheng, W., Davidsen, A. F., \& Tytler, D. 2002, ApJ, 579, 500

Tenorio-Tagle, G. 1996, AJ, 111, 1641

Thuan, T. X. \& Martin, G. E. 1981, ApJ, 247, 823

Thuan, T. X. \& Izotov, Y. I. 2005, ApJS, 161, 240

Thuan, T. X. \& Izotov, Y. I. 2005b, ApJ, 627, 739

Thuan, T. X., Izotov, Y. I., \& Lipovetsky, V.A. 1997, ApJ, 477, 661

Thuan, T. X., Sauvage, M., \& Madden, S. 1999, ApJ, 516, 783

Thuan, T. X., Lecavelier des Etangs, A., \& Izotov, Y. I. 2005, ApJ, 621, 269

Thuan, T. X., Hunt, L. K., \& Izotov, Y. I. 2008, ApJ, 689,

Thuan, T. X., Lipovetsky, V. A., Martin, J.-M., \& Pustilnik, S. A. 1999b, A\&ASS, 139, 1 
Thuan, T. X., Bauer, F. E, Papaderos, P., \& Izotov, Y. I. 2004, ApJ, 606, 213

van Zee, L., Westpfahl, D., Haynes, M., \& Salzer, J. 1998, AJ, 115, 1000

Vanzi, L., Hunt, L. K., Thuan, T. X., \& Izotov, Y. I. 2000, A\&SA, 363, 493

Vidal-Madjar, A., et al. 2000, ApJ, 538, L77

Wu, Y., Charmandaris, V., Hao, L., et al. 2006, ApJ, 639, 157

Wu, Y., Charmandaris, V., Hunt, L. K., et al. 2007, ApJ, 662, 952

Zwicky, F. 1971, Catalogue of Selected Compact Galaxies and of Post Eruptive Galaxies, Bern, Switzerland 